\title{
Estimación del límite de fatiga de carburos cementados WC-Co en el marco de la mecánica de la fractura elástica lineal
}

\author{
Y. TORRES, M. ANGLADA, L. LLANES \\ Departament de Ciència dels Materials i Enginyería Metal-lúrgica, ETSEIB, Universitat Politècnica de Catalunya, 08028 Barcelona
}

\begin{abstract}
En este trabajo se investiga el comportamiento a fractura y fatiga de dos grados de carburos cementados WC-Co con distintos contenidos de fase ligante y tamaño de carburo medio. La caracterización mecánica incluye la evaluación de la resistencia a flexión, la tenacidad de fractura, el límite de fatiga y la cinética de propagación de grietas grandes por fatiga (PGGF) para relaciones de carga diferentes. Se propone un enfoque basado en la mecánica de la fractura elástica lineal con el objetivo de evaluar la relación existente entre la vida a fatiga y la PGGF. El análisis se concentra en la correlación límite de fatiga-umbral de propagación de grietas para condiciones de vida a fatiga infinita. Así, se estima el límite de fatiga asociado con defectos naturales a partir del umbral de propagación experimentalmente determinado para grietas grandes, asumiendo que: (1) existe similitud en el comportamiento a fatiga de fisuras grandes y pequeñas, y (2) los defectos críticos bajo cargas monotónicas y cíclicas son los mismos. La fiabilidad de este enfoque para evaluar condiciones límites bajo solicitaciones cíclicas se sustenta en la concordancia satisfactoria observada para los valores del límite de fatiga estimados y los experimentalmente determinados para las distintas razones de carga investigadas.
\end{abstract}

Palabras clave: Propagación de grietas por fatiga, límite de fatiga, carburos cementados, mecánica de fractura

\section{Fatigue limit estimation for WC-Co cemented carbides on the basis of linear elastic fracture mechanics}

The fracture and fatigue behavior of two microstructurally different hardmetals (WC-Co cemented carbides) is investigated. Mechanical characterization includes flexural strength and fracture toughness as well as fatigue limit and fatigue crack growth (FCG) behavior under monotonic and cyclic loads respectively. Considering that fatigue lifetime of cemented carbides is given by subcritical crack growth of preexisting defects, a linear elastic fracture mechanics approach is attempted to assess fatigue life - FCG relationships for these materials. It concentrates on addressing the fatigue limit - FCG threshold correlation under infinite fatigue life conditions. Thus, the fatigue limit associated with natural flaws is estimated from FCG threshold experimentally determined for large cracks under the assumptions that (1) similitude on the FCG behavior of small and large cracks applies for cemented carbides, and (2) critical flaws are the same, in terms of nature, geometry and size, under monotonic and cyclic loading. The reliability of this fatigue mechanics approach is sustained through the satisfactory agreement observed between estimated and experimentally determined values for the fatigue limit under the different load ratios investigated.

Keywords: Fatigue crack growth, fatigue limit, fracture mechanics, cemented carbides

\section{INTRODUCCIÓN}

La susceptibilidad de los carburos cementados a la degradación mecánica bajo cargas cíclicas es un hecho conocido desde hace más de medio siglo (1). Sin embargo, la información existente sobre las propiedades de fatiga de estos materiales puede considerarse escasa, una vez que se compara con la publicada sobre su comportamiento a fractura [ver por ejemplo Refs. $(2,3)]$. Sólo en años recientes y como resultado de la investigación extensa y bien estructurada efectuada por Sockel y col. (4-8), el comportamiento a fatiga de los carburos cementados parece haber recibido su justo reconocimiento como factor crítico en el diseño de herramientas y componentes fabricados con estos materiales. En general, la evaluación de la vida a fatiga de WCCo ha sido la línea de la mayoría de las investigaciones en esta área (4-10), siendo pocos los estudios enfocados a la propagación de fisuras por fatiga (10-12). Sin embargo, en ninguna de las investigaciones se ha intentado correlacionar la resistencia a la propagación de grietas grandes por fatiga (PGGF) y la vida a fatiga de estos materiales.

En el presente trabajo se pretende alcanzar una mejor comprensión de los fenómenos de fatiga que ocurren en el metal duro. Asimismo, considerando el planteamiento establecido de que la vida a fatiga de estos materiales viene dictada por el crecimiento subcrítico de grietas a partir de los defectos intrínsecos preexistentes en los mismos, en esta investigación se utiliza un enfoque basado en la mecánica de la fractura elástica lineal (MFEL) para analizar la correlación entre el límite de fatiga y el umbral de PGGF para condiciones de vida a fatiga infinita. Para ello, el tamaño del defecto crítico bajo cargas cíclicas es definido simplemente en términos del umbral de fatiga. En este marco de ideas, los valores del límite de fatiga para los grados de carburos cementados WC-Co estudiados se estiman a partir de las medidas experimentales de la tenacidad de fractura y la PGGF, asumiendo que: 1) existe similitud en el comportamiento a fatiga de fisuras grandes y pequeñas en estos materiales, y 2) los defectos críticos obtenidos bajo cargas monotónicas y cíclicas son los mismos en términos de naturaleza, geometría y tamaño.

\section{PROCEDIMIENTO EXPERIMENTAL}

En este trabajo se estudian dos grados de carburos cementados con distintos contenidos de cobalto y tamaño de carburo medio. En la nomenclatura utilizada para definirlos (16F y 27C) el número contabiliza el contenido en volumen de la fase ligante $\left(\mathrm{V}_{\mathrm{CO}_{0}}\right)$, mientras que la letra se refiere al tamaño de la fase cerámica $\left(\mathrm{d}_{\mathrm{wC}}\right)$ en términos de fino y grueso respectivamente. La caracterización mecánica llevada a cabo incluye la evaluación de la resistencia a la rotura transversal, $\sigma_{\mathrm{R}^{\prime}}$ la tenacidad de fractura, $\mathrm{K}_{\mathrm{Ic}}$ el límite de fatiga, $\Delta \sigma_{\mathrm{f}^{\prime}}$ y de la PGGF. 
El estudio de la $\mathrm{K}_{\mathrm{Ic}}$ y la PGGF requiere de la prefisuración del material. En este trabajo se realizó mediante la aplicación de cargas cíclicas de compresión a probetas prismáticas entalladas, cuyas dimensiones fueron de $45 \times 10 \times 5 \mathrm{~mm}$. Es ampliamente reconocido la efectividad de este método para generar fisuras agudas en materiales frágiles, aunque induce tensiones residuales de tracción (13). En esta investigación, con el objetivo de eliminar el efecto de estas tensiones, todas las prefisuras se propagaron adicionalmente $1 \mathrm{~mm}$ aproximadamente (bajo fatiga a tracción) hasta alcanzar la zona no afectada. Para evaluar la $\mathrm{K}_{\mathrm{Ic}}$ se utilizaron cuatro muestras. Las mismas se ensayaron a flexión en cuatro puntos hasta la rotura, con una separación entre puntos de apoyo exteriores e interiores de 40 y $20 \mathrm{~mm}$ respectivamente. Por su parte, el estudio de la PGGF se realizó mediante la aplicación de cargas cíclicas de tracción. Para llevar a cabo dichos experimentos se utilizó una máquina servohidráulica con célula de carga de $20 \mathrm{kN}$ y control digital. Se aplicaron cocientes de carga de 0,1, 0,4 y 0,7. Estos ensayos se realizaron a frecuencias entre 0,5 y $10 \mathrm{~Hz}$, a temperatura ambiente y humedad relativa del $40 \%$. El crecimiento de las fisuras se evaluó mediante dos métodos complementarios. Uno de seguimiento continuo donde se utilizó un microscopio óptico de larga distancia focal a 1000 aumentos y otro de seguimiento discontinuo donde se empleó otro microscopio óptico, y para lo cual las muestras tuvieron que ser desmontadas del sistema de ensayo.

En lo que concierne a la evaluación de $\sigma_{\mathrm{R}}$ y $\Delta \sigma_{f^{\prime}}$ estos parámetros se determinaron utilizando probetas prismáticas con dimensiones de $45 \times 4 \times 3 \mathrm{~mm}$, a las cuales además del desbaste y pulido de la cara que se sometió a tracción, se les eliminó sus bordes para evitar de esta forma posibles concentradores de tensión. En estos estudios se empleó la misma configuración del ensayo de flexión en cuatro puntos descrita anteriormente. Para evaluar la $\sigma_{\mathrm{R}}$ se utilizaron seis probetas y se aplicó una velocidad de carga de $100 \mathrm{~N} / \mathrm{s}$. El estudio del $\Delta \sigma_{f}$ (definido para una vida a fatiga mayor que $10^{7}$ ciclos) se realizó siguiendo el método de ensayo de la escalera (staircase) (14). Este método se implementó en el caso del grado $16 \mathrm{~F}$ para las tres relaciones de carga investigadas. En todos los casos se ensayaron 17 muestras como mínimo en una máquina de resonancia a una frecuencia de $170 \mathrm{~Hz}$ aproximadamente.

Las superficies de fractura se analizaron, para todas las muestras estudiadas, a través de un examen minucioso mediante microscopía electrónica de barrido MEB. Para cada probeta, los posibles sitios de inicio de la fractura se encontraron inicialmente a un aumento bajo. Posteriormente, estas áreas se examinaron a un aumento mayor siendo así posible observar, medir y discernir el tamaño, la geometría y el tipo de defecto que originó la fractura bajo las distintas solicitaciones impuestas (monotónicas y cíclicas).

\section{RESULTADOS Y DISCUSIÓN}

\subsection{Dureza, resistencia mecánica y tenacidad de fractura}

Los resultados de dureza Vickers (HV30), $\sigma_{\mathrm{R}}$ y $\mathrm{K}_{\mathrm{Ic}}$ se muestran en la tabla I. En la literatura está bien establecido que la dureza es inversamente proporcional a la tenacidad de fractura y que disminuye tanto al aumentar el tamaño de los carburos para un contenido de cobalto constante como al incrementar el contenido de cobalto para un tamaño de grano constante. Sin embargo, cuando se varían a la vez el contenido de ligante y el tamaño de carburo medio, es imposible establecer relaciones simples entre estos parámetros microestructurales y las propiedades mecánicas. Este inconveniente puede ser eliminado si se utiliza el camino libre medio de la fase metálica, $\lambda_{\mathrm{C}^{\prime}}$ como parámetro normalizador de la microestructura de los metales duros. Éste es una medida del tamaño de la fase ligante y en él se contabiliza a la vez la influencia tanto del contenido de cobalto como del tamaño de carburo medio. Un aumento del $\lambda_{\mathrm{Co}}$ se traduce en un incremento de la ductilidad efectiva del ligante metálico constreñido por los carburos de wolframio. En general, se observa que la dureza disminuye y la tenacidad aumenta a medida que el $\lambda_{\mathrm{Co}}$ es mayor.

TABla I. Características microestructurales $\left(\mathrm{V}_{\mathrm{Co}^{\prime}} \mathrm{d}_{\mathrm{WC}} \mathrm{y} \lambda_{\mathrm{Co}}\right)$ y PROPIEDADES MECÁNICAS (HV30, $\sigma_{\mathrm{R}}$ y $\mathrm{K}_{\mathrm{Ic}}$ ) PARA LOS GRADOS DE CARBUROS CEMENTADOS WC-CO ESTUDIADOS EN ESTE TRABAJO.

\begin{tabular}{|c|c|c|c|c|c|c|}
\hline Grado & $\mathbf{V}_{\mathrm{co}}(\%)$ & $\mathbf{d}_{\mathrm{wC}}(\mu \mathbf{m})$ & $\lambda_{\mathrm{co}}(\mu \mathbf{m})$ & $\mathbf{H V 3 0}(\mathbf{G P a})$ & $\sigma_{\mathrm{R}}(\mathbf{M P a})$ & $\left.\mathbf{K}_{\mathrm{Ic}} \mathbf{M P a m}^{1 / 2}\right)$ \\
\hline $\mathbf{1 6 F}$ & $16,30 \pm 0,02$ & 0,50 & 0,25 & $15,4 \pm 2,5$ & $2742 \pm 130$ & $9,2 \pm 0,6$ \\
\hline $\mathbf{2 7 C}$ & $27,38 \pm 0,07$ & 1,66 & 0,76 & $11,2 \pm 0,8$ & $2629 \pm 241$ & $14,7 \pm 1,0$ \\
\hline
\end{tabular}

\subsection{Caracterización de la cinética de PGGF: efecto de $R$ y sensibilidad a fatiga}

En la figura 1 se muestra la velocidad de PGGF en función de $\Delta \mathrm{K}$ para los materiales y relaciones de carga estudiados. El comportamiento encontrado es bien conocido para estos materiales $(10-12,15)$, y se puede resumir según las siguientes observaciones: 1) la extensión de las grietas comienza para valores de $\Delta \mathrm{K}$ mucho más bajos que aquellos donde $\mathrm{K}_{\max }$ tiende a $\mathrm{K}_{\mathrm{Ic}^{\prime}}$ 2) existe una dependencia marcada, siguiendo una ley potencial, entre da/dN y $\Delta \mathrm{K}$, con pendientes que decrecen tanto cuando el camino libre medio de la fase ligante aumenta, como cuando disminuye la relación de carga aplicada, y 3) la influencia de la relación de carga es significativa, siendo mayor a medida que disminuye el camino libre medio de la fase ligante.
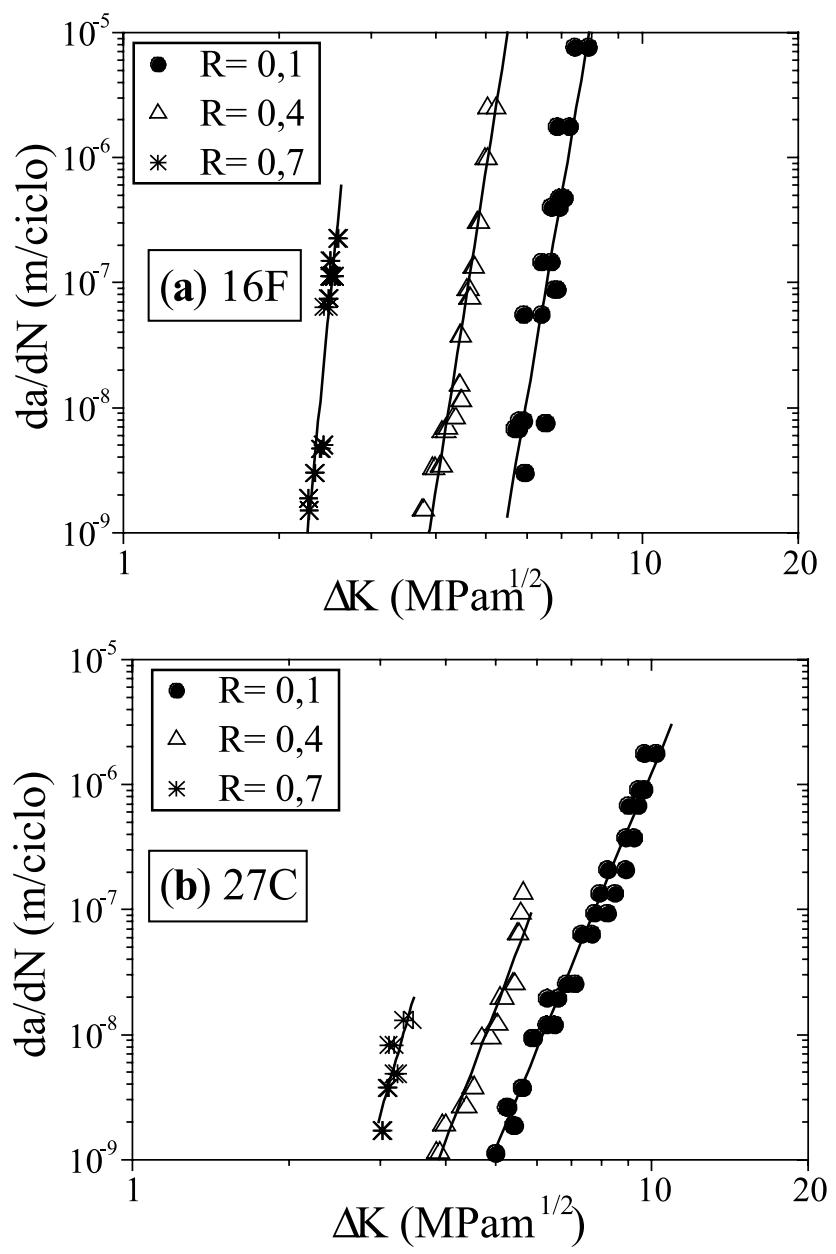

Figura 1. Cinética de PGGF para diferentes valores de R: a) 16F, y b) 27C. 
En la tabla II se resumen, para cada material y condición de ensayo, los valores de $\Delta K_{\text {th }}$ definidos a partir del valor de $\Delta K$ para el cual la cinética de propagación de fisuras es del orden de $10^{-9} \mathrm{~m} /$ ciclo. Por debajo de estos valores, aunque basado en un número relativamente escaso de datos debido a las limitaciones experimentales implícitas a su evaluación, se pudo discernir un cambio en la pendiente de la cinética de crecimiento de fisuras con el factor de intensidad de tensiones a valores aún más extremos que los ya determinados en la etapa de PGGF estable, indicando de esta forma la transición al régimen umbral. Por su parte, en esta investigación se define la sensibilidad a fatiga como $\Delta \mathrm{K}_{\mathrm{th}} / \mathrm{K}_{\mathrm{Ic}}$ observándose por un lado una relación directa entre ésta y el camino libre medio de cobalto independientemente de la relación de carga aplicada y por el otro, un incremento en la influencia de la microestructura a medida que $\mathrm{R}$ disminuye.

TABLA II. VALORES DEL UMBRAL DEL RANGO DEL FACTOR DE INTENSIDAD DE TENSIONES ASOCIADO A LA PGGF EN LOS MATERIALES Y CONDICIONES INVESTIGADAS.

\begin{tabular}{|c|c|c|c|}
\hline & \multicolumn{3}{|c|}{$\Delta \mathbf{K}_{\text {th }}\left(\mathbf{M P a m}^{\mathbf{1} / 2}\right)$} \\
\hline & $\mathbf{R}=\mathbf{0 , 1}$ & $\mathbf{R}=\mathbf{0}, \mathbf{4}$ & $\mathbf{R}=\mathbf{0 , 7}$ \\
\hline $\mathbf{1 6 F}$ & 5,40 & 3,84 & 2,25 \\
\hline $\mathbf{2 7 C}$ & 4,86 & 3,90 & 2,82 \\
\hline
\end{tabular}

\subsection{Determinación del límite de fatiga}

En la figura 2 se muestra en detalle la secuencia completa del ensayo de la escalera para $\mathrm{R}=0,1$ en el grado $16 \mathrm{~F}$. Luego de procesar estadísticamente los resultados obtenidos con este método, se determinó $\Delta \sigma_{\mathrm{f}}$ con un $95 \%$ de confianza para cada una de las condiciones y materiales analizados. Los límites de fatiga estimados (ver más detalles en el próximo apartado) y determinados experimentalmente se resumen en la tabla III. En todos los casos, los resultados se expresan en términos del rango del esfuerzo aplicado, $\Delta \sigma$.

TABLA III. VALORES DE LOS LÍMITES DE FATIGA, EN TÉRMINOS DEL RANGO DEL ESFUERZO APLICADO PARA LOS DIFERENTES MATERIALES Y RELACIONES DE CARGAS ESTUDIADAS.

\begin{tabular}{|c|c|c|c|c|c|c|}
\hline & \multicolumn{5}{|c|}{ Límite de fatiga (MPa) } \\
\hline Grado & \multicolumn{2}{|c|}{$\mathbf{R = 0 , 1}$} & \multicolumn{2}{c|}{$\mathbf{R = 0 , 4}$} & \multicolumn{2}{c|}{$\mathbf{R}=\mathbf{0 , 7}$} \\
\hline & Experimental & Estimado & Experimental & Estimado & Experimental & Estimado \\
\hline 16F & $1644 \pm 92$ & 1609 & $1070 \pm 70$ & 1162 & $634 \pm 70$ & 680 \\
\hline 27C & - & 869 & - & 697 & - & 504 \\
\hline
\end{tabular}

\subsection{Correlación entre el límite de fatiga y el umbral de PGGF}

En este apartado se evalúa la relación entre la PGGF y el $\Delta \sigma_{f^{\prime}}$ lo cual permitiría optimizar el diseño apropiado y la selección del material a utilizar en aplicaciones que involucran fatiga. Aunque esta relación podría intentarse siguiendo una metodología de tolerancia de daño, ello no parece ser una ruta conveniente ni fácil. El comentario anterior se sustenta en las enormes incertidumbres que existen en una predicción asociada a la fuerte dependencia de la cinética de PGGF con $\Delta K$, como ocurre con los carburos cementados WC-Co (figura 1). En cambio, una aproximación más clásica y conservadora sobre las bases del $\Delta \sigma_{\mathrm{f}}$ y el $\Delta \mathrm{K}_{\mathrm{th}}$ parece ser más apropiada para estos materiales. En este trabajo, esta aproximación se implementa definiendo simplemente el tamaño del defecto crítico bajo cargas cíclicas en términos del umbral de PGGF. Bajo esta consideración, el límite de fatiga vendría dado por

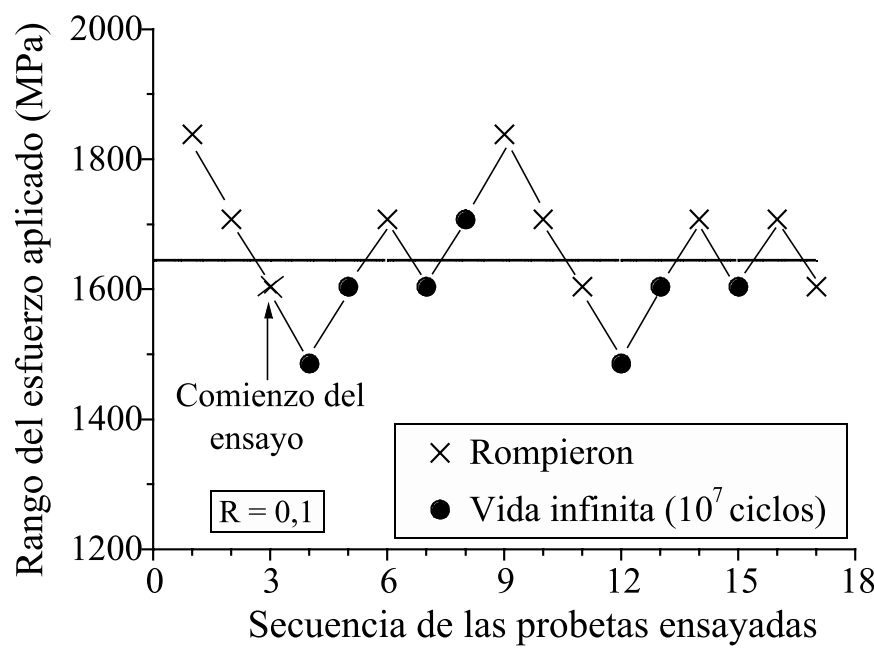

Figura 2. Secuencia completa del ensayo siguiendo el método de la escalera para $\mathrm{R}=0,1$ en el grado $16 \mathrm{~F}$.

el $\Delta \sigma$ resultante en el $\Delta \mathrm{K}_{\text {th }}$ de una fisura pequeña que emana a partir de un defecto de tamaño crítico, $2 \mathrm{a}_{\mathrm{c}^{1}}$ según relaciones del tipo:

$$
\Delta \sigma_{\mathrm{f}} \propto \frac{\Delta \mathrm{K}_{\mathrm{th}}}{\sqrt{\mathrm{a}_{\mathrm{cr}}}}
$$

Entonces, los valores del $\Delta \sigma_{\mathrm{f}}$ podrían estimarse a partir de los resultados experimentales presentados en las secciones anteriores mediante la correlación fundamental de la MFEL entre el tamaño del defecto, la resistencia y las condiciones umbrales evaluadas para grietas grandes, asumiendo que éstas últimas son válidas también para defectos naturales. Es ampliamente conocido que en muchos materiales metálicos y cerámicos avanzados la propagación de fisuras pequeñas por fatiga difiere considerablemente de la mostrada por fisuras grandes, particularmente en la zona por debajo del $\Delta \mathrm{K}_{\text {th }}$ [por ejemplo, ver Ref. (16)]. Sin embargo, en los carburos cementados no se espera que ocurra este comportamiento anómalo. Esta afirmación se sustenta en que: 1) el tamaño de los defectos naturales (de procesado) es varias veces mayor que el tamaño de carburo medio (unidad microestructural de estos materiales), 2) la zona plástica que se genera delante de la punta de la grieta se encuentra confinada en la zona de proceso y su extensión es menor que $\lambda_{\text {Co }}$ y que el tamaño de los defectos preexistentes $(17,18), y$ 3) la zona de multiligamentos formada detrás de la punta de la fisura es del orden de cinco carburos $(17,18)$, esperándose que las diferencias en la propagación de grietas grandes y pequeñas no sea significativa. Así, el límite de fatiga puede ser estimado directamente a partir de la siguiente expresión:

$$
\Delta \sigma_{\mathrm{f}}=\left(\frac{\Delta \mathrm{K}_{\mathrm{th}}}{\mathrm{K}_{\mathrm{Ic}}}\right) \sigma_{\mathrm{R}}
$$

partiendo de la hipótesis de que los defectos que controlan la fractura tanto bajo cargas monotónicas como cíclicas son los mismos en cuanto al tipo, geometría, tamaño, y distribución. Esta aproximación encuentra soporte experimental en el examen fractográfico minucioso llevado a cabo (por ejemplo, figura 3). Además, la correlación entre el $\Delta \sigma_{\mathrm{f}}$ estimado a partir de la ecuación 2 y el obtenido experimentalmente (ver tabla III) mediante el método de la escalera es satisfactoria. Estos dos aspectos experimentales sustentan y corroboran la fiabilidad del enfoque planteado en esta investigación. En otras palabras, se puede predecir directamente el límite de fatiga de carburos cementados mediante la sensibilidad a fatiga $\left(\Delta \mathrm{K}_{\mathrm{th}} / \mathrm{K}_{\mathrm{lc}}\right)$ evaluada a partir de la PGGF en ellos. 

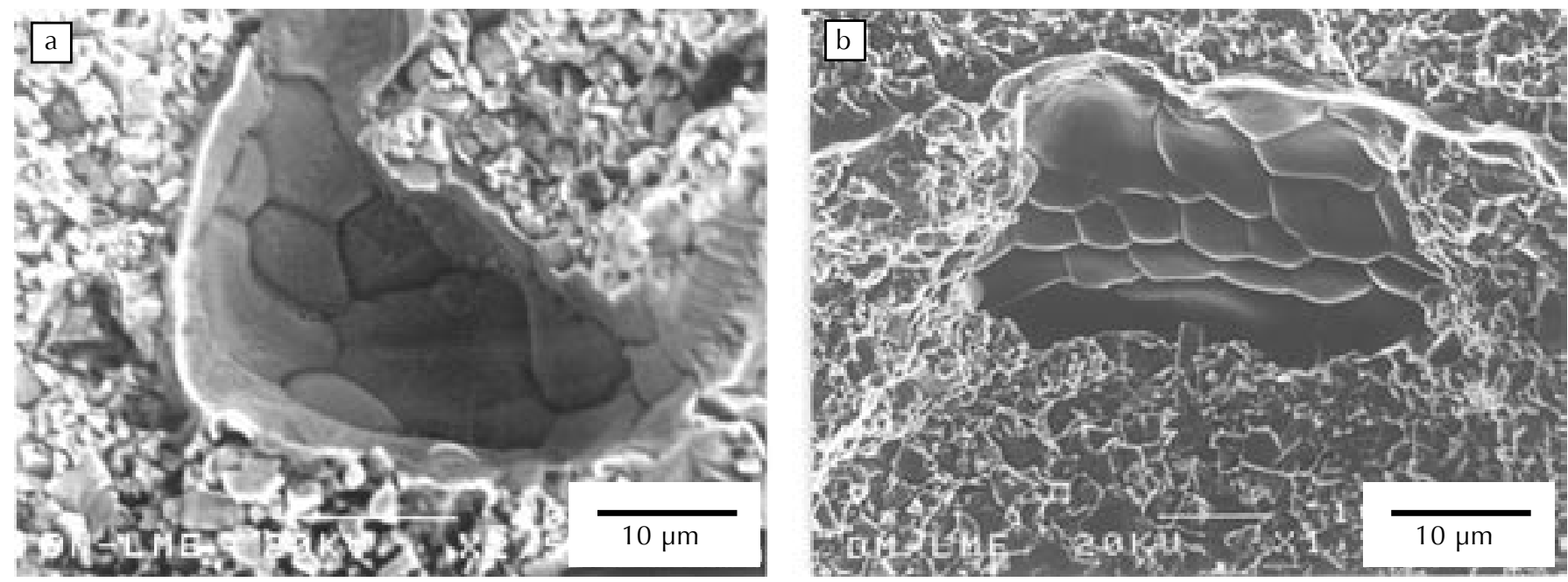

Figura 3. Defectos típicos (poros) a partir de los cuales se origina la fractura en el material 27C: a) cargas monotónicas y b) cargas cíclicas.

\section{CONCLUSIONES}

Los resultados y las conclusiones principales a las que se han arribado en este trabajo se resumen a continuación:

1) El comportamiento a fractura (tenacidad de fractura) y fatiga (cinética de PGGF y sensibilidad a fatiga) de los carburos cementados WC-Co varía significativamente con la microestructura de estos materiales. Tal influencia puede ser racionalizada utilizando el camino libre medio de cobalto, observándose conductas diferentes en presencia de cargas monotónicas y cíclicas (forma y magnitud) a medida que este parámetro aumenta, es decir, al incrementar la ductilidad efectiva de la fase metálica constreñida.

2) El límite de fatiga de carburos cementados WC-Co puede ser racionalizado satisfactoriamente mediante un análisis en el marco de la MFEL. Esta afirmación es válida considerando el crecimiento subcrítico de fisuras como la etapa que controla la rotura por fatiga, es decir, definiendo el $\Delta \mathrm{K}_{\text {th }}$ como la tenacidad efectiva bajo cargas cíclicas.

\section{AGRADECIMIENTOS}

Los autores agradecen el soporte financiero proporcionado por la Comisión Interministerial de Ciencia y Tecnología (CICYT) en el marco del proyecto No MAT2000-1014-C02-01. Los autores agradecen especialmente la colaboración de M. Marsal en el análisis por MEB.

\section{BIBLIOGRAFÍA}

1. W. Dawihl. «Die wissenschaftlichen und technischen grundlagen der pulvermetallurgie». Stahl u. Eisen. 61 909-919 (1941).

2. H.F. Fischmeister. «Development and present status of the science and technology of hard materials», pp. 1-45 en Science of Hard Materials. Ed. R. K. Viswanatham, D. J. Rowcliffe, J. Gurland, Plenum Press, New York (EE.UU.) (1984).
3. B. Roebuck, E.A. Almond. «Deformation and fracture processes and the physical metallurgy of WC-Co hardmetals». Int. Mater. Rev. 33 90-110 (1988).

4. U. Schleinkofer, H.G. Sockel, P. Schlund, K. Görting, W. Heinrich. «Behaviour of hard metals and cermets under cyclic mechanical loads». Mater. Sci. Eng. A194 1-8 (1995)

5. U. Schleinkofer, H.G. Sockel, K. Görting, W. Heinrich. «Microstructural processes during subcritical crack growth in hard metals and cermets under cyclic loads». Mater. Sci. Eng. A209 103-110 (1996).

6. U. Schleinkofer, H.G. Sockel, K. Görting, W. Heinrich. «Fatigue of hard metals and cermets». Mater. Sci. Eng. A209 313-317 (1996).

7. U. Schleinkofer, H.G. Sockel, K. Görting, W. Heinrich. «Fatigue of hard metals and cermets - new results and a better understanding». Int. J. Refract. Met. Hard Mater. 15 103-112 (1997).

8. Th. Sailer, M. Herr, H.G. Sockel, R. Schulte, H. Feld, L.J. Prakash. «Microstructure and mechanical properties of ultrafine-grained hardmetals». Int. J. Refract. Met. Hard Mater. 19 553-559 (2001).

9. F.-C. Dary, B. Roebuck, M.G. Gee. «Effects of microstructure on the thermomechanical fatigue response of hardmetals using a new miniaturized testing ring». Int. J. Refract. Met. Hard Mater. 17 45-53 (1999).

10. S. Ishihara, T. Goshima, T. Yoshimoto, T. Sabu. «On fatigue lifetimes and crack growth behavior of cemented carbides», pp. 1811-1816 en Fatigue'99 vol 3: Proc. 7th Int. Fatigue Congress, Beijing (China), Ed. X.R. Wu, Z.G. Wang, (1999).

11. E.A. Almond, B. Roebuck. «Fatigue-crack growth in WC-Co hardmetals». Met. Technol. 2 83-85 (1980).

12. P.R. Fry, G.G. Garret. «The inter-relation of microstructure toughness and fatigue crack growth behaviour in WC-Co hardmetals», pp. 375-381 en Proc. Int. on Speciality Steels and Hard Materials. Ed. N.P. Comins, J.B. Clark, Pergamon, London (U.K.) (1983).

13. M.N. James, A.M. Human, S. Luyckx. «Fracture toughness testing of hardmetals using compression-compression precracking». J. Mater. Sci. 25 4810-4814 (1990).

14. Collins, J. A., «Failure of materials in mechanical design». J. Wiley \& Sons, New York (EE.UU.) 369-374 (1981).

15. Y. Hirose, M.-H. Boo, H. Matsuoka, Y.-C. Park. «Influence of stress ratio and WC grain size on fatigue crack growth characteristics of WC-Co cemented carbides». J. Soc. Mat. Sci. Japan. 46 1402-1409 (1997).

16. R.O. Ritchie. «Mechanisms of fatigue crack propagation in metals, ceramics and composites: role of crack tip shielding». Mater. Sci. Eng. 103 15-28 (1988).

17. L.S. Sigl, H.E. Exner. «Experimental study of the mechanics of fracture in WC-Co alloys». Metall. Trans. A. 18 1299-1308 (1987).

18. L.S. Sigl, H.F. Fischmeister. «On the fracture toughness of cemented carbides». Acta Metall. 36 887-897 (1988).

Recibido: 1.2.03

Aceptado: 30.11 .03 